\title{
Repetition priming from moving faces
}

\author{
KAREN LANDER \\ University of Manchester, Manchester, England \\ and \\ VICKI BRUCE \\ University of Edinburgh, Edinburgh, Scotland
}

\begin{abstract}
Recent experiments have suggested that seeing a familiar face move provides additional dynamic information to the viewer, useful in the recognition of identity. In four experiments, repetition priming was used to investigate whether dynamic information is intrinsic to the underlying face representations. The results suggest that a moving image primes more effectively than a static image, even when the same static image is shown in the prime and the test phases (Experiment 1). Furthermore, when moving images are presented in the test phase (Experiment 2), there is an advantage for moving prime images. The most priming advantage is found with naturally moving faces, rather than with those shown in slow motion (Experiment 3). Finally, showing the same moving sequence at prime and test produced more priming than that found when different moving sequences were shown (Experiment 4). The results suggest that dynamic information is intrinsic to the face representations and that there is an advantage to viewing the same moving sequence at prime and test.
\end{abstract}

In most experiments on face recognition, static images of faces (e.g., photographs or slides) have been used as stimuli. However, recent experiments have shown that seeing a familiar face move aids the recognition of identity. Knight and Johnston (1997) first demonstrated this effect by showing that famous faces presented in negative (contrastreversed) format were better recognized when shown moving than when they were shown as a single static image. No significant advantage of motion was found when the same images were presented upside down (inverted), whether in a negative or a positive (monochrome) format. Knight and Johnston proposed that seeing the face move may provide evidence about its three-dimensional structure, compensating for the degraded depth cues within a negative image (see Bruce \& Langton, 1994). Alternatively, Knight and Johnston suggested that known faces may have characteristic facial gestures, idiosyncratic to the individual viewed.

More recent follow-ups to this work (see Lander, Bruce, \& Hill, 2001; Lander, Christie, \& Bruce, 1999) have shown that the recognition advantage for moving faces is not specific to negated images. Instead, motion confers benefits through a range of image manipulations, including inversion, thresholding (whereby a multiple a gray-level image is converted to a one-bit-per-pixel black-and-white image),

This work was supported by a University of Stirling PhD studentship awarded to K.L. and by ESRC Grant R000222989 awarded to V.B. and K.L. The authors thank Michael Lewis, Yonatan Goshen-Gottstein, and an anonymous reviewer for their helpful comments on an earlier draft of this article. Correspondence concerning this article should be addressed to K. Lander, Department of Psychology, University of Manchester, Oxford Road, Manchester M13 9PL, England (e-mail: karen.lander@ psy.man.ac.uk) pixelation, and Gaussian blurring. Spatially degrading the spatial information in this manner is needed to reduce recognition performance below ceiling levels, maximizing participants' reliance on motion-based cues. Of course, this finding does not preclude any role for motion when effective static information is available; rather, any such benefit is difficult to demonstrate. In these experiments, the moving sequences involve famous faces talking and expressing (nonrigid motion), with some limited rigid motion of the head (nodding and shaking). It seems clear that under a range of nonoptimum viewing conditions, motion of the internal face features has the ability to add information useful for recognition.

Importantly, it has been shown (see Lander \& Bruce, 2000; Lander et al., 1999) that the recognition advantage for moving images is not due solely to the increased amount of static-based information contained in a moving sequence. Indeed, when the same frames were shown but not animated, recognition rates were significantly lower than when they were shown as an animated sequence (Lander et al., 1999). Furthermore, the precise dynamic characteristics of the observed motion were important in mediating the recognition advantage of motion. Recognition of thresholded images was better from sequences that maintained their original dynamic properties than with the same sequences shown speeded up, slowed down, with the rhythm disrupted, or in reverse (Lander \& Bruce, 2000; Lander et al., 1999). Thus, seeing a face moving "naturally" (where the original dynamic characteristics of the motion are preserved) provided the greatest advantage for recognition.

Further evidence that viewers are tuned to precise patterns of observed motion comes from work by Hill and Johnston (2001), who computer-animated an average head 
with movements captured from real people, to investigate whether motion alone can provide useful information for categorizing identity and sex (sex decision experiments not described here). The movements of 12 volunteers, telling a joke, were used to animate the same three-dimensional head model. The participants were presented with three animations - two examples of one person and one example of a different person - and were asked to identify the odd one out. Animations were presented normally (upright, forward motion), backward, or with the faces upside down. The results indicated that performance was above chance and that people can discriminate between individuals on the basis of motion-based information alone.

In another ingenious study, Knappmeyer, Thornton, and Bülthoff (2001) exposed participants to two synthetic heads, each animated by the movement captured from a different volunteer (Face A animated by Motion A, Face B animated by Motion B). In a later testing phase, the participants viewed an animated head, constructed from a morph of the two synthetic heads (a head that was structurally midway between Face A and Face B) and were asked to identify who was shown. The results showed that the participants' identity judgments were biased by the motion information originally learned. Thus, there are a number of studies that suggest that dynamic information, provided by seeing a face move, can support the recognition process.

O'Toole, Roark, and Abdi (2002) speculated that seeing the face move may enhance the perception of the static structure of the face, which in turn is helpful for recognition (representational enhancement hypothesis). Such an effect would not depend on having prior experience with a face. Instead, seeing a face move would provide a richer or more complete structural description of a face, relative to a static image (Knight \& Johnston, 1997). However, this interpretation is not easy to reconcile with studies in which benefits of motion have not been found in recognition memory experiments in which unfamiliar faces were used (Christie \& Bruce, 1998).

Alternatively, individual faces may have characteristic motion signatures, which capture the idiosyncratic aspects of their facial movement patterns (termed supplemental information hypothesis by O'Toole et al., 2002). Here, experience with the face is needed to learn an individual's characteristic motion style. Such characteristic motion information may be intrinsic to the stored face identity representations (cf. dynamic representations; Freyd, 1987, 1993) or may provide the viewer with an independent additional source of information (extrinsic to face representation). We explored these possibilities, using repetition priming. Repetition priming is the facilitation demonstrated at test when the to-be-recognized item has been previously encountered at some time prior to test. Such effects have been reported for words (e.g., Jackson \& Morton, 1984) and objects (e.g., Bruce, Carson, Burton, \& Ellis, 2000; Warren \& Morton, 1982), as well as for familiar faces (e.g., Bruce \& Valentine, 1985). A number of recent articles have shown different priming effects for moving and static objects across image changes and temporal delays (Kourtzi \& Nakayama, 2002; see also Kourtzi \& Shriffrar, 2001, for further work on object priming).

In recent years, many researchers have used repetition priming to probe the nature of the representations underlying recognition (but see Warren \& Morton, 1982). Indeed, Biederman and Cooper (1992) claimed that repetition priming provides a more appropriate tool for probing the representations of objects than do matching or memory tasks. Biederman and Cooper $(1991,1992)$ found more priming when the same exemplars of an object were shown at prime and test (e.g., upright piano-upright piano), provided that each exemplar showed the same visual object components (geons), as compared with different exemplars (e.g., upright piano-grand piano). They assumed that because both views presented at prime access the same conceptual and verbal information about the object, it is only the difference between same and different exemplars that taps visual priming. Thus, the two exemplars do not access a common visual representation.

Applying this argument to faces, when priming is sensitive to some change in the form of the faces between study and test, this dimension may be intrinsic to the representations mediating face recognition (see also Cooper, Schacter, Ballesteros, \& Moore, 1992; Kalocsai \& Biederman, 1997; Schacter, Cooper, \& Delaney, 1990). In later work, Fiser and Biederman (2001), on the basis of a series of repetition priming experiments, concluded that the visual object representations are insensitive to low-level variations in an image (spatial frequency or the precise location or orientation of object features) that do not alter the basic part-structure of the object. However, for faces, Bruce, Burton, Carson, Hanna, and Mason (1994) concluded that low-level visual information is preserved in the representations, since reduced priming is shown when there is a change in the format of an image between prime and test (e.g., cartoon-photo rather than cartoon-cartoon). In this article, we compare the amount of priming from moving and static faces, using our findings to draw conclusions about the nature of the underlying face representations. More specifically, we investigate whether moving faces promote more repetition priming than do static primes. Previous studies have shown that repetition priming is maximal when the same face image is shown at prime and test (see, e.g., Warren \& Morton, 1982), with less but still significant priming when a different image of the same person is viewed (Ellis, Burton, Young, \& Flude, 1997; Ellis, Young, Flude, \& Hay, 1987). No studies, to our knowledge, have compared the amount of priming from moving and static faces.

In Experiment 1, we compared the amount of repetition priming from a moving prime image with that from a static prime image. In this experiment, the images in the test phase were always static. In the static condition, exactly the same image was presented in the prime and the test phases of the experiment. Interestingly, we found that moving images primed more effectively than static ones. In Experiment 2, we used the same methodology and compared the 
amount of priming from moving and static images for moving test sequences (same moving sequence viewed at prime and test). Again, viewing a moving face in the prime phase promoted the most priming. In Experiment 3, we investigated whether the robust priming effect was linked to the motion by comparing the amount of priming from moving, slow motion, and static prime images (static at test). The most priming advantage was found with naturally moving faces, rather than with those shown in slow motion (Experiment 3). Finally, in Experiment 4, we compared the amount of priming when the same moving sequence was shown at prime and test. Viewing the same sequence at prime and test produced more priming than that found when different moving sequences were shown (Experiment 4).

\section{EXPERIMENT 1}

\section{Method}

Participants. Twenty-four undergraduate students at the University of Stirling took part in the experiment. Each was paid for participating. None had taken part in any previous priming experiments or took part in any of the other experiments reported here.

Materials. A set of moving video images consisting of 32 famous faces was collected by digitizing images from video (Media 100 software). Images in the prime phase were presented to the participant on a Sony television, using VHS video. The presentation time of the moving images was set to $2,500 \mathrm{msec}$. All the images filled the $30 \times 21 \mathrm{~cm}$ presentation screen. All of the moving clips displayed at least the head and shoulders of the person from a frontal viewpoint. Since the images were taken from television productions, some people were shown from the waist upward. The movement displayed was mainly of a nonrigid nature (expressions and speech), with some limited rigid motion of the head and rotation about the waist. The static images were created by selecting a single freeze frame from the moving sequence. In all cases, the moving images were compressed (using cinepak compression) before display, whereas the static images were uncompressed. Thus, any impairment in the static condition was unlikely to reflect a difference in image quality that might have resulted from image grabbing.

In the static condition, the first frame (Image A) of the moving clip was selected as the static image (shown for 2,500 msec). The static images were typical of the moving sequence, in that they avoided any unusual momentary expressions or head angles.

In the test phase, the stimuli were presented on a computer monitor (Macintosh Performa 630), using Superlab software. All the images viewed in the test phase were static and were sized $12 \mathrm{~cm}$ long $X$ $9 \mathrm{~cm}$ wide, although the size of the face varied for different identities. The size of the face on the screen varied in width from 3.1 to $7.2 \mathrm{~cm}$ (average width $=4.6 \mathrm{~cm}$ ) and in height from 4.2 to $7.9 \mathrm{~cm}$ (average height $=5.1 \mathrm{~cm}$ ). Thus, at a viewing distance of $50 \mathrm{~cm}$, the face was displayed within an average region of $5.2^{\circ}$ in width $\times 5.8^{\circ}$ in height. For each of the famous faces, the first frame of the moving sequence was shown in the test phase (Image A). Thus, in the static same condition, the same static image was shown in the prime and the test phases.

Design. The experiment had one within-subjects factor of prime condition (images presented moving, static, and unprimed). The dependent measure was response time (RT) in the test phase.

Procedure. The experiment consisted of a priming phase and a test phase. The two experimental phases were presented to the participants as separate experiments, with an unrelated experiment carried out between the two phases. The minimum and maximum times between the appearance of a prime stimulus and its reoccurrence in the test phase were between 16 and $25 \mathrm{~min}$. This procedure is similar to that employed in a large number of studies of face repetition priming (see Brunas, Young, \& Ellis, 1990; Ellis et al., 1987).

In the prime phase, the participants were shown a series of famous faces and were tested individually. They were asked to name or provide some semantic information about the person displayed. Names of roles played (e.g., "Alexis Carrington" for Joan Collins) were deemed correct, as were unambiguous descriptions of the person (e.g., "Prime Minister" for Tony Blair). General information, such as "actor" or "comedian" in the absence of any further information, was deemed not sufficient for a correct response. Each face was followed by a 5 -sec interstimulus interval (black screen shown), during which the participants were asked to respond verbally with their answers. No feedback was given to the participants.

During the prime phase, all the participants viewed both moving and static faces. The 30 famous faces selected were split into three groups of 10 faces. The participants were presented with two of the three groups ( 20 famous faces) in the prime phase of the experiment; one group consisted of moving images, and the other group consisted of static images (static same conditions). The remaining group formed the unprimed condition (faces not shown). The experiment was a repeated measures design, and the three groups of faces were counterbalanced across conditions so that each face appeared in each experimental condition to different participants. The order of presentation in the prime phase was also randomized.

In the test phase of the experiment, the participants viewed 76 face images of different people, arranged in a fixed random order. They were asked to make a speeded familiarity judgment by manually pressing one of two keys on the computer keyboard. It is important to note that the participants were simply required to judge whether each face in the test phase was familiar or unfamiliar: They were not required to judge whether the face had been viewed in the earlier prime phase. After a decision had been made, there was a brief pause $(200 \mathrm{msec})$ before the next image was displayed.

Of the 76 faces viewed in the test phase, 38 belonged to famous people, 20 of which had previously been viewed by the participants in the prime phase (10 in the moving condition and 10 in the static same condition) and 10 of which made up the unprimed condition. The remaining 8 famous faces made up a filler group, which had not been viewed in the prime phase and were discounted from any further analysis. These filler famous faces were included in order to reduce the percentage of primed trials and, so, to minimize possible episodic memory effects. The remaining 38 faces shown in the test phase were of unfamiliar people. The unfamiliar face images were created in exactly the same way as the famous face images. A single freeze frame was selected from a moving sequence, which was originally digitized from television.

Six faces (three familiar and three unfamiliar) were presented at the beginning of the test phase during a practice sequence, to ensure that the participants knew the task requirements. The results from these practice trials were not analyzed further.

\section{Results and Discussion}

Recognition performance (hits) in the prime phase of the experiment was high (moving condition, 93\%; static same condition, $87 \%$ ), indicating that the famous faces used in the experiment were highly familiar to the participants taking part in the experiment. A related $t$ test revealed that the difference between recognition in the moving and the static conditions of the prime phase just reached significance $[t(23)=1.83, p>.05$, one-tailed test $]$.

The response time data from the test phase were analyzed both by participants and by items, using a one-way analysis of variance (ANOVA), with prime condition as the factor. If a face was not correctly identified in the prime phase, the response to that face for that particular participant was 
Table 1

Mean Reaction Times (RTs)

With Standard Deviations (SDs) and Percentages of Error (\%E) in Each of the Conditions in Experiments 1-4

\begin{tabular}{|c|c|c|c|}
\hline \multirow[b]{2}{*}{ Condition } & \multicolumn{2}{|c|}{ RT } & \\
\hline & $M$ & $S D$ & $\% \mathrm{E}$ \\
\hline
\end{tabular}

Experiment 1 (Static Images at Test)

$\begin{array}{llll}\text { Moving } & 733 & 139 & 0.5 \\ \text { Static } & 781 & 157 & 2.0 \\ \text { Unprimed } & 907 & 205 & 7.0\end{array}$

Experiment 2 (Moving Images at Test)

$\begin{array}{llll}\text { Moving } & 724 & 170 & 0.5 \\ \text { Static } & 769 & 167 & 1.0 \\ \text { Unprimed } & 840 & 208 & 1.5\end{array}$

Experiment 3 (Static Images at Test)

$\begin{array}{lllr}\text { Moving } & 746 & 160 & 2.0 \\ \text { Slowed } & 791 & 162 & 2.0 \\ \text { Static } & 816 & 232 & 1.0 \\ \text { Unprimed } & 892 & 240 & 12.0\end{array}$

Experiment 4 (Moving Same Images at Test)

$\begin{array}{llll}\text { Moving same } & 718 & 100 & 1.0 \\ \text { Moving different } & 749 & 122 & 2.0 \\ \text { Static } & 781 & 107 & 2.0 \\ \text { Unprimed } & 828 & 159 & 9.0\end{array}$

disregarded. All the participants correctly recognized at least 14 faces of the 20 displayed in the prime phase. RTs to familiar faces judged to be unfamiliar in the test phase (errors) were also eliminated from the analysis. The participants made few errors in the familiarity decision task, and error rates did not differ significantly across primed conditions. Means and error rates across participants are presented in Table 1.

The effect of prime condition was significant by participants $\left[F(2,46)=33.18, M S_{\mathrm{e}}=5,819.82\right]$ and by items $\left[F(2,58)=18.98, M S_{\mathrm{e}}=13,181.95, p<.001\right]$. Post hoc Newman-Keuls pairwise comparisons on both the byparticipants and the by-items means revealed, as was expected, that there was a significant difference between the unprimed and both of the primed conditions. Thus, familiarity judgments were significantly enhanced by prior exposure to the same face. This finding is in line with a large number of previous studies that have demonstrated repetition priming for familiar faces (e.g., Bruce \& Valentine, 1985; Brunas et al., 1990; Ellis et al., 1987). Interestingly, familiarity decisions in the moving condition were significantly faster than those in the static condition.

Before turning to a more detailed investigation of this robust repetition priming from moving images, we investigated the amount of priming shown when moving images are presented at test. It was important to establish that the advantage for moving primes found in Experiment 1 was not linked to the format of the test phase but, rather, can be found with both moving and static test images.

\section{EXPERIMENT 2}

\section{Method}

The design and procedure in this experiment were the same as those in the previous experiment, except for the following details.
Participants. Twenty-four undergraduate students at the University of Stirling took part in the experiment. None took part in any of the other experiments reported here.

Materials. The stimuli used in this experiment were the same as those in Experiment 1, except that in this experiment, all the images, in both the prime and the test phases, were presented to the participant on a computer (G4 AppleMac), using PsyScope software (see Cohen, MacWhinney, Flatt, \& Provost, 1993). Digitized moving images were converted to QuickTime movies, using Cinepak compression. All the static images were exported from the digital video editing software (Media 100) as pict images, without any compression.

Design. The experiment had one within-subjects factor of prime condition (images presented moving, static, or unprimed). As in the previous experiment, the static image consisted of the first frame of the moving sequence, shown for $2,500 \mathrm{msec}$. All the images in the test phase were presented as moving sequences.

Procedure. The 30 famous faces were split into three groups of 10 faces. The participants were presented with two of the three groups (20 famous faces) in the prime phase of the experiment, one group in the moving condition and one in the static condition. The remaining group formed the unprimed condition. Faces were counterbalanced across conditions so that all the faces were seen in all the conditions across participants. The participants were asked to name or provide some unambiguous semantic information about each person viewed.

In the test phase, the participants were asked to make a speeded familiarity decision by manually pressing a key. When the participant had made a choice, the moving image stopped and disappeared from the screen. RT was measured from when the moving image started to when the participant indicated a familiarity decision by pressing a key. If the participant failed to make a choice within the 2,500-msec moving image, the last frame of the moving sequence remained on the screen until a decision had been made. There was then a short break $(250 \mathrm{msec})$ before the next image was displayed. Thirty-eight faces belonged to famous people, 20 that had previously been viewed by the participant in the prime phase and 10 that made up the unprimed condition. The remaining 8 famous faces made up a filler group, which had not been viewed in the prime phase. The remaining 38 faces shown in the test phase were of unfamiliar people.

\section{Results and Discussion}

Recognition performance in the prime phase of the experiment was high (moving condition, $90 \%$; static condition, 86\%). A related $t$ test revealed that the difference in recognition between the moving and the static conditions in the prime phase did not reach significance $[t(23)=$ $1.36, p>.05]$.

The RT data from the test phase were analyzed both by participants and by items, using a one-way ANOVA with prime condition as the factor. Means and error rates across participants are presented in Table 1 . The analyses revealed a significant effect of prime condition by participants $\left[F(2,46)=30.57, M S_{\mathrm{e}}=2,690.30, p<.001\right]$ and by items $\left[F(2,58)=13.01, M S_{\mathrm{e}}=7,313.59, p<.001\right]$. A Newman-Keuls a posteriori test of pairwise comparisons on both the by-participants and the by-items means revealed the expected difference between the unprimed condition and both primed conditions. As was expected, familiarity decisions in the moving prime condition were significantly faster than those in the static prime condition. It seems that viewing a moving image in the prime phase leads to significantly more priming, regardless of whether the test image is moving or static. In the remain- 
der of this article, we will explore possible reasons for this advantage.

One possible explanation of the beneficial effect of motion (as compared with a static image) is that a moving sequence contains more static-based information (more instances) than does a static image. A moving sequence potentially provides multiple triggers to the recognition unit, because of the different viewpoints and expressions it contains. If so, multiple viewpoint presentations should increase priming over a single frame event. However, previous repetition priming experiments have confirmed that there is no advantage to viewing multiple presentations of the same face during a recognition epoch (e.g., A-blankB-blank-C-blank-D), as compared with viewing the same image presented repeatedly (A-blank-A-blankA-blank-A; see Lander, 1999). This finding suggests that changing viewpoints, by showing multiple images within the same recognition epoch, is not the sole reason why moving sequences are so effective as primes.

Previous explicit recognition experiments have suggested that identity is better recognized from a moving image, predominantly because of the additional dynamic information afforded by motion. If the robust priming effect found with moving images is due to additional dynamic information, altering the dynamic characteristics of the observed motion should affect the amount of priming. To test this suggestion, in Experiment 3 we compared the amount of priming from moving, slow motion, and static primes. By slowing down the tempo of the observed motion, we alter the original dynamic characteristics of the observed motion. If the motion is intrinsic to the representations underlying face recognition, we might expect the most benefit to be derived from naturally moving faces. However, even altered motion (as in the slowed condition) may be better than none at all (static condition). Thus, we might expect less but still significant priming from slow motion primes, as compared with naturally moving clips.

An alternative interpretation is that the moving images promote more priming, not because motion is intrinsic to the underlying representations, but because more attention is paid to them. According to this interpretation, there is likely to be significant priming from both naturally moving and slow motion primes, but not in different amounts. Indeed, it is difficult to consider why more attention would be paid to a naturally moving face than to one shown in slow motion.

To summarize, if motion is intrinsic to the face representations, we would expect the most priming from naturally moving faces, as compared with that from those shown in slow motion.

\section{EXPERIMENT 3}

\section{Method}

The design and procedure for this experiment were the same as those detailed in previous experiments, except for the following details.

Participants. Forty undergraduate students at the University of Stirling took part in the experiment. None took part in any other of the experiments reported here.
Materials. The stimuli used in this experiment were created from moving video clips collected for previous experiments. In this experiment, the moving sequence was shortened to $1,500 \mathrm{msec}$ (rather than $2,500 \mathrm{msec}$ ) and was played four times in succession (total display time $=6,000 \mathrm{msec}$ ). In other work (Lander, 1999), we confirmed that there is no advantage from viewing multiple presentations of the same image during a recognition epoch (A-blank-A-blank-Ablank-A), as compared with the same image shown continuously (AAAA). Thus, we have confirmed that there is no advantage for multiple presentations of the same face.

The slowed-down clips were created by decreasing the frame rate by a factor of four. Consequently, the slowed-down clips were four times the duration of the original moving clips (total display time $=$ $6,000 \mathrm{msec}$ ). This meant that the moving and the slowed-down clips showed exactly the same selection and number of images for the same amount of time. The original dynamic characteristics of the motion were preserved in the moving sequence but were removed in the slowed condition. As before, the static condition consisted of the first frame of the moving sequence (Image A). The static image was displayed for $6,000 \mathrm{msec}$ in the prime phase.

In the test phase of this experiment, the same static images were used, but they were edited to remove the background and clothing from the images. This ensured that our effects were due to the faces, rather than to explicit memory for context. All the face images were depicted against the same medium gray background and were sized to fit comfortably within a circular $7.5-\mathrm{cm}$ background.

Design. The experiment had one within-subjects factor of prime condition (images presented moving, slowed, static, or unprimed).

Procedure. The 40 famous faces were split into four groups (10 faces each). The participants were presented with three of the four groups ( 30 famous faces) in the prime phase of the experiment, one group in the moving condition, one in the slowed condition, and one in the static condition. The remaining group formed the unprimed condition. Faces were counterbalanced across conditions so that all the faces were seen in all the conditions across participants. In the prime phase, all the images were displayed on television, using a video. The participants were asked to name or provide some unambiguous semantic information about each person viewed.

In the test phase, they were asked to make a speeded familiarity decision by manually pressing a key. Forty-eight faces belonged to famous people, 30 of which had previously been viewed by the participant in the prime phase (10 in the moving condition, 10 in the slowed condition, and 10 in the static condition), and 10 made up the unprimed condition. The remaining 8 famous faces made up a filler group, which had not been viewed in the prime phase and were discounted from any further analysis. The remaining 48 faces shown in the test phase were of unfamiliar people.

\section{Results and Discussion}

Recognition performance in the prime phase of the experiment was high (moving condition, 90\%; slowed condition, $86 \%$; static condition, $85 \%$ ). A one-way ANOVA revealed that there was no significant difference in recognition rates across conditions in the prime phase $[F(2,78)=$ $\left.1.46, M S_{\mathrm{e}}=79.76, p>.05\right]$.

The RT data from the test phase were analyzed both by participants and by items, using a one-way ANOVA, with prime condition as the factor. Means and error rates across participants are presented in Table 1 . The analyses revealed a significant effect of prime condition by participants $\left[F(3,117)=20.86, M S_{\mathrm{e}}=7,103.27, p<.001\right]$ and by items $\left[F(3,117)=8.80, M S_{\mathrm{e}}=16,596.54, p<.001\right]$. A Newman-Keuls a posteriori test of pairwise comparisons on both the by-participants and the by-items means revealed the expected difference between the unprimed 
condition and all the primed conditions. RTs in the moving condition were significantly faster than those in both the slowed and the static conditions, which did not differ. Thus, it is likely that the advantage of moving primes is due to the additional dynamic information afforded by motion and that changing the dynamic parameters by slowing the tempo reduced the amount of priming. If the participants were simply paying more attention to moving faces, we would expect to find similar amounts of priming from both naturally moving and slow motion primes; we found no support for this suggestion.

Interestingly, there was no significant difference between the amounts of priming from faces shown in slow motion and those shown as single static images. Previous explicit recognition experiments indicated that the addition of any type of motion aids recognition, although natural motion is of the most benefit (see Lander \& Bruce, 2000). We may have failed to find this difference here because of the small size of these priming effects. Furthermore, the images used in this experiment were nondegraded. In the experiments detailed in Lander and Bruce, we used thresholded images, which may have forced the participants to rely more heavily on dynamic parameters as a cue to identity.

So we have found the most priming from naturally moving faces, as compared with those shown in slow motion, implying that motion is intrinsic to the underlying face representations. In our final experiment, we explored whether there would be any advantage for viewing the same moving sequence at prime and test, as compared with viewing a different moving sequence.

\section{EXPERIMENT 4}

\section{Method}

The design and procedure for this experiment were the same as those detailed in the previous experiments, except for the following details.

Participants. Forty undergraduate students at the University of Manchester took part in the experiment. None had taken part in any other of the experiments reported here.

Materials. The moving clips used in previous experiments were again used in this experiment. Furthermore, we created a new set of moving images of the same famous faces. These were selected from the same run of original video, thus ensuring that background and context remained constant across the two moving clips of one person. We were careful to make sure that the moving clips did not overlap in terms of the selection of frames. We refer to these moving clips as moving same and moving different. Thus, the participants viewed both moving-same and moving-different clips at prime (showing different faces). The static image was a freeze frame selected from the moving-same clip. The test phase of this experiment used the moving-same clip.

Design. The experiment had one within-subjects factor of prime condition (images presented moving same, moving different, static, or unprimed).

Procedure. The 40 famous faces were split into four groups of 10 faces each. The participants were presented with three of the four groups (30 famous faces) in the prime phase of the experiment, one group in the moving-same condition, one in the moving-different condition, and one in the static condition. The remaining group formed the unprimed condition. Faces were counterbalanced across conditions so that all the faces were seen in all the conditions across participants. In the prime phase, all the images were displayed on computer (G4 PowerMac), using PsyScope software (Cohen et al., 1993). The participants were asked to name or provide some unambiguous semantic information about each person viewed.

In the test phase, they were asked to make a speeded familiarity decision by manually pressing a key. Forty-eight faces belonged to famous people, 30 that had previously been viewed by the participant in the prime phase (10 in the moving-same condition, 10 in the moving-different condition, and 10 in the static condition), and 10 made up the unprimed condition. The remaining 8 famous faces made up a filler group, which had not been viewed in the prime phase and were discounted from any further analysis. The remaining 48 faces shown in the test phase were of unfamiliar people.

\section{Results and Discussion}

Recognition performance in the prime phase of the experiment was high (moving-same condition, 91\%; movingdifferent condition, $90 \%$; static condition, $87 \%$ ). A one-way ANOVA revealed that there was no significant difference in recognition rates across conditions in the prime phase $\left[F(2,78)=3.06, M S_{\mathrm{e}}=60.77, p>.05\right]$.

The RT data from the test phase were analyzed both by participants and by items, using a one-way ANOVA, with prime condition as the factor. Means and error rates across participants are presented in Table 1 . The analyses revealed a significant effect of prime condition by participants $\left[F(3,117)=17.59, M S_{\mathrm{e}}=4,989.65, p<.05\right]$ and by items $\left[F(3,117)=8.4, M S_{\mathrm{e}}=16,982.48, p<.001\right]$. A Newman-Keuls a posteriori test of pairwise comparisons on both the by-participants and the by-items means revealed the expected difference between the unprimed condition and all the primed conditions. RTs in the movingsame condition were significantly faster than those in the moving-different condition. RTs in both of the moving conditions were significantly faster than those in the static condition. It seems, then, that the most priming is shown when the same moving sequence is shown at prime and test, implying that overlap is also an important factor in determining the amount of priming.

\section{GENERAL DISCUSSION}

The four experiments reported here allow us to make the following points. First and most centrally, we have demonstrated that moving images prime significantly more than static ones, even when the same static image is shown in the prime and the test phases of the experiment (Experiment 1). Such differences do not seem to simply reflect the fact that moving faces were processed better or that moving faces were recognized spontaneously more often than static faces in the prime phase. ${ }^{1}$ Before considering the implications of the robust priming by moving faces, we discounted the possibility that these effects were linked to the format of the test images, so that it could be concluded that they reflected differences of image presentation in the prime phase. ${ }^{2}$ Experiment 2 explored this possibility by using moving images at test. The results indicated that the most priming was found when the same moving sequence was presented at prime and test, as compared with a sin- 
gle static prime image. Thus, we suggest that viewing a moving image in the prime phase leads to significantly more priming, regardless of the test image format.

Interestingly, our robust priming effect with moving images appears to be related to the additional dynamic information afforded by motion. We speculated that if the dynamic characteristics of the observed motion were important in mediating the robust priming by moving images, altering these characteristics should adversely affect the amount of priming. Experiment 3 showed the most priming from naturally moving primes, as compared with those shown in slow motion. ${ }^{3}$ It is suggested that dynamic information is somehow intrinsic to the stored face representations. Additional work is needed to distinguish exactly how dynamic information is incorporated into face representations. One possibility is that the stored face representations are themselves dynamic in nature (see Freyd, 1987). If the representations mediating face recognition are dynamic (whereby the temporal dimension is inextricably embedded in the representation; see Freyd \& Pantzer, 1995), recognition from a static image should be thought of as a "snapshot" within an essentially dynamic process.

Finally, in Experiment 4, we compared the amount of priming when the same moving sequence was shown at prime and test with that for a different moving sequence selected from the same run of video. The results showed that there was an advantage for viewing the same moving sequence at prime and test, although both moving prime conditions promoted more priming than did the static condition.

So how do we explain these results with reference to current models of repetition priming of faces? And what are the implications of our findings? Our results pose challenges for all current theories of repetition priming. Perhaps the best developed account of repetition priming outlined in the literature is the structural interactive activation and competition (IAC) account proposed by Burton and colleagues (Burton, Bruce, \& Hancock, 1999; Burton, Bruce, \& Johnston, 1990). IAC suggests that repetition priming arises as a function of the strengthening of connections within the face recognition system. In order to explain our findings with moving faces, we would need some way to represent the features of a moving face, without which no such attempt can be made to simulate the results. This may require exploring the effects of coding of image sequences, using principal components analysis or other techniques (see, e.g., Bartlett \& Sejnowski, 1998, McKenna \& Gong, 1998) and developing ideas about how representations of familiar faces can be derived from such sequences. However, the IAC account would appear to be challenged by any findings that show more priming from something that is less like the original trigger. Similar problems arise, in principle, from other models of repetition priming that emphasize overlap between prime and test conditions or overlap between procedures to be critical. The main issue we highlight is that the representational bases of most models are simply not developed well enough at present. The present findings emphasize the central role that dynamic features should play in these elaborated models of the future.

\section{REFERENCES}

Bartlett, M. S., \& Sejnowski, T. J. (1998). Learning viewpointinvariant face representations from visual experience in an attractor network. Network Computation in Neural Systems, 9, 399-417.

Biederman, I., \& Cooper, E. E. (1991). Evidence for complete translational and reflectional invariance in visual object priming. Perception, 20, 585-593.

BiEDERMAN, I., \& COOPER, E. E. (1992). Size invariance in visual object priming. Journal of Experimental Psychology: Human Perception \& Performance, 18, 121-133.

BRUCE, V. (1982). Changing faces: Visual and nonvisual coding processes in face recognition. British Journal of Psychology, 73, 105-116.

Bruce, V., Burton, A. M., Carson, D., Hanna, E., \& Mason, O. (1994). Repetition priming of face recognition. In C. Umiltà \& M. Moscovitsch (Eds.), Attention and performance XV: Conscious and nonconscious information processing (pp. 179-201). Cambridge, MA: MIT Press, Bradford Books.

Bruce, V., Carson, D., Burton, M. A., \& Ellis, A. W. (2000). Perceptual priming is not a necessary consequence of semantic classification of pictures. Quarterly Journal of Experimental Psychology, 53A, 289-323.

BRUCE, V., \& LANGTON, S. (1994). The use of pigmentation and shading information in recognising the sex and identities of faces. Perception, 23, 803-822.

BRUCE, V., \& VALENTINE, T. (1985). Identity priming in the recognition of familiar faces. British Journal of Psychology, 76, 373-383.

Brunas, J., Young, A. W., \& Ellis, A. W. (1990). Repetition priming from incomplete faces: Evidence for part to whole completion. British Journal of Psychology, 81, 43-56.

Burton, A. M., Bruce, V., \& HanCock, P. (1999). From pixels to people: A model of familiar face recognition. Cognitive Science, 23, 1-31.

Burton, A. M., Bruce, V., \& Johnston, R. A. (1990). Understanding face recognition with an interactive activation model. British Journal of Psychology, 81, 361-380.

CHRISTIE, F., \& BRUCE, V. (1998). The role of dynamic information in the recognition of unfamiliar faces. Memory \& Cognition, 26, 780-790.

Cohen, J., MacWhinney, B., Flatt, M., \& Provost, J. (1993). PsyScope: An interactive graphic system for designing and controlling experiments in the psychology laboratory using Macintosh computers. Behavior Research Methods, Instruments, \& Computers, 25, 257-271.

Cooper, L. A., Schacter, D. L., Ballesteros, S., \& Moore, C. (1992). Priming and recognition of transformed three-dimensional objects: Effects of size and reflection. Journal of Experimental Psychology: Learning, Memory, \& Cognition, 18, 43-57.

Ellis, A. W., Burton, A. M., Young, A., \& Flude, B. M. (1997). Repetition priming between parts and wholes: Tests of a computational model of familiar face recognition. British Journal of Psychology, $\mathbf{8 8}$, 579-608.

Ellis, A. W., Young, A. W., Flude, B. M., \& Hay, D. C. (1987). Repetition priming of face recognition. Quarterly Journal of Experimental Psychology, 39A, 193-210.

FISER, J., \& BIEDERMAN, I. (2001). Invariance of long-term visual priming to scale, reflection, translation and hemisphere. Vision Research, 41, 221-234.

FREYD, J. J. (1987). Dynamic mental representations. Psychological Review, 94, 427-438.

FREYD, J. J. (1993). Five hunches about perceptual processes and dynamic representations. In D. Meyer \& S. Kornblum (Eds.), Attention and performance XIV: Synergies in experimental psychology, artificial intelligence, and cognitive neuroscience (pp. 99-120). Cambridge, MA: MIT Press.

Freyd, J. J., \& PANTzer, T. M. (1995). Static patterns moving in the mind. In S. S. Smith, T. B. Ward, \& R. A. Finke (Eds.), The creative cognition approach (pp. 181-204). Cambridge, MA: MIT Press.

Hill, H., \& Johnston, A. (2001). Categorizing sex and identity from the biological motion of faces. Current Biology, 11, 880-885. 
JACKSON, A., \& MORTON, J. (1984). Facilitation of auditory word recognition. Memory \& Cognition, 12, 568-574.

Johnston, R. A., \& BARRY, C. (2001). Best face forward: Similarity effects in repetition priming of face recognition. Quarterly Journal of Experimental Psychology, 54A, 383-396.

KalocsaI, P., \& Biederman, I. (1997). Difference of face and object recognition in utilizing early visual information. In $\mathrm{H}$. Wechsler, P. J. Phillips, V. Bruce, F. Fogelman Soulié, \& T. S. Huang (Eds.), Face recognition: From theory to applications (Nato ASI series: Series F. Computer and systems sciences: Vol. 163). Berlin: Springer-Verlag.

KNAPPMEYer, B., Thornton, I., \& BÜlthoff, H. H. (2001). Facial motion can determine facial identity [Abstract]. Journal of Vision, 1, 338a.

Knight, B., \& Johnston, A. (1997). The role of movement in face recognition. Visual Cognition, 4, 265-273.

KourTZI, Z., \& NAKAYAMA, K. (2002). Distinct mechanisms for the representation of moving and static objects. Visual Cognition, 9, 248-264.

KourtZi, Z., \& Shriffrar, M. (2001). Visual representation of malleable and rigid objects as they rotate. Journal of Experimental Psychology: Human Perception \& Performance, 27, 335-355.

LANDER, K. (1999). The role of dynamic information in the recognition of famous faces. Unpublished $\mathrm{PhD}$ thesis, University of Stirling.

LANDER, K., \& BRUCE, V. (2000). Recognising famous faces: Exploring the benefits of facial motion. Ecological Psychology, 12, 259-272.

LANDER, K., BRUCE, V., \& HiLl, H. (2001). Evaluating the effectiveness of pixelation and blurring to mask the identity of familiar faces. $A p$ plied Cognitive Psychology, 15, 101-116.

LANDER, K., Christie, F., \& BRUCE, V. (1999). The role of movement in the recognition of famous faces. Memory \& Cognition, 27, 974-985.

McKenna, S. J., \& Gong, S. (1998). Real-time pose estimation. RealTime Imaging, 4, 333-347.

O'Toole, A. J., RoArK, D.A., \& ABdi, H. (2002). Recognizing moving faces: A psychological and neural synthesis. Trends in Cognitive Sciences, 6, 261-266.

Schacter, D. I., Cooper, L. A., \& Delaney, S. M. (1990). Implicit memory for unfamiliar objects depends on access to structural descriptions. Journal of Experimental Psychology: General, 119, 5-24.

WARREN, C., \& MorTON, J. (1982). The effects of priming on picture recognition. British Journal of Psychology, 73, 117-130.

\section{NOTES}

1. The prime phase difference between recognition of moving and static faces (moving better) in Experiment 1 reached significance. However, in all the other experiments reported, we found no significant difference in recognition in the prime phase. Indeed, the amount of priming does not seem to clearly reflect the amount of processing at prime. For example, in related work (unpublished), we compared the amount of priming from gray-scale and thresholded static prime images for thresholded static images at test. Although gray-scale images were recognized significantly better at prime [gray-scale $=87 \%$, thresholded $=77 \%$; $t(17)=2.76, p<.05]$, there was more priming from thresholded prime images [gray-scale mean $=876 \mathrm{msec}$, thresholded mean $=821 \mathrm{msec}$, unprimed $\left.=1,166 \mathrm{msec} ; F(2,34)=44.67, M S_{\mathrm{e}}=13,640.32, p<.05\right]$. Similarly, in published work, Johnston and Barry (2001) found that the amount of priming was not related to the goodness of the prime face image (Experiment 1), even though "good likeness" images were recognized much better at the prime stage, as compared with poor likenesses (3.8\% unrecognized at prime, as compared with $12.5 \%$; figures taken from the article).

2. In the experiments reported, we demonstrated more priming from moving primes than from static ones, even though the same static image was presented at test. Such a result would not be predicted by conscious recollection. Indeed, in early work (see Bruce, 1982), recognition memory for familiar faces was adversely affected by changes in view. In line with this prediction, we have recently run a control experiment comparing an implicit repetition priming experiment with an explicit recognition memory version of this experiment. Both experiments proceeded in the same manner, with participants ( 20 per experiment) being asked to name or provide semantic information about moving and static famous faces in Part 1 of the experiment. Overall, recognition rates were high in Part 1 of the experiment, with no significant difference between moving and static conditions in either the explicit or the implicit version of the experiment [implicit experiment moving condition $=91 \%$, static condition $=85 \%, t(19)=1.48, p>.05$; explicit experiment moving condition $=91 \%$, static condition $86 \%, t(19)=1.31, p>.05]$. In Part 2 of the experiment, the implicit repetition priming participants were asked "Is this face familiar?" (yes/no; RT measured), whereas the explicit recognition memory participants were asked "Did this face appear in the first part of this experiment?" (yes/no; RT measured). The results replicated the robust priming effect found with moving faces, with significantly more priming from moving primes than from static ones [20 participants; mean moving RT $=658 \mathrm{msec}$, mean static RT $=711 \mathrm{msec}$, mean unprimed RT $=836 \mathrm{msec} ; F(2,38)=25.28, M S_{\mathrm{e}}=6,645.22, p<$ $.05]$. However, in the explicit version of the experiment, the participants were significantly faster to respond "yes" (face has been viewed in Part 1 of this experiment) to static than to moving faces [20 participants; mean moving RT $=755 \mathrm{msec}$, mean static RT $=711 \mathrm{msec} ; t(19)=4.75, p<$ $.05]$. Thus, the results reported in this article cannot be explained by reference to conscious recollection.

3. In follow-up student work (unpublished data), we have compared the amount of priming from moving, speeded-up, and static prime images. If slowed-down images do not grab attention (due to their being perceived in the same way as static images), we might expect speeded images to be more attention grabbing. The results again suggest that the most priming is found from naturally moving prime sequences $[18$ participants; moving $=725 \mathrm{msec}$, speeded-up $=770 \mathrm{msec}$, static $=813 \mathrm{msec}$, unprimed $\left.=895 \mathrm{msec} ; F(3,51)=18.3, M S_{\mathrm{e}}=5,141.78, p<.05\right]$.

(Manuscript received October 15, 2001; revision accepted for publication November 27, 2003.) 